\title{
Impacts of Study Area Spatial Form on Co-location Quotient Analysis: Evidence From Medical Resources Distribution in Wuhan City
}

\section{Qiao Chen}

School of Tourism and Hospitality Management, Hubei University of Economics

Jianquan Cheng

Department of Natural Sciences, Manchester Metropolitan University

Jianguang Tu ( $\nabla$ tu.jianguang@whu.edu.cn )

School of Remote Sensing Information Engineering, Wuhan University

\section{Research Article}

Keywords: Medical resource distribution, health GIS, localised colocation quotient, study area spatial form, multi-scale, Wuhan

Posted Date: November 9th, 2021

DOI: https://doi.org/10.21203/rs.3.rs-1020355/v1

License: (c) (i) This work is licensed under a Creative Commons Attribution 4.0 International License. Read Full License 


\title{
Impacts of study area spatial form on co-location quotient analysis: evidence from medical resources distribution in Wuhan city
}

\author{
Qiao Chen ${ }^{1}$, Jianquan Cheng ${ }^{2,3}$ and Jianguang $\mathrm{Tu}^{4,5 *}$
}

\begin{abstract}
School of Tourism and Hospitality Management, Hubei University of Economics, Wuhan 430205, China; chenqiao@ hbue.edu.cn Department of Natural Sciences, Manchester Metropolitan University, Manchester, M1 5GD, UK; J.Cheng@mmu.ac.uk

3 Centre for Health Geographic Information, Key Laboratory of Environmental Change and Resource Use in Beibu Gulf (Ministry of Education), Nanning Normal University, 175 MingxiuDonglu Road, 530051, Nanning, PR China;

4 School of Remote Sensing Information Engineering, Wuhan University, 430070, Wuhan, PR.China;

5 China Aero Geophysical Survey and Remote Sensing Center for Natural Resource, 100083, Beijing;

* Correspondence: tu.jianguang@whu.edu.cn;
\end{abstract}

\begin{abstract}
Background: There is a sharp contradiction between supply and demand of medical resources in provincial capitals of China. Understanding the spatial patterns of medical resources and identifying their spatial association and heterogeneity is a prerequisite to ensuring limited resources are allocated fairly and optimally, which, along with improvements to urban residents' quality of life, is a key aim of healthy city planning.

Method: Localised co-location quotient (LCLQ) analysis has been used successfully to measure directional spatial associations and heterogeneity between categorical point data. Using point of interest data and the LCLQ method, this paper presents the first analysis of spatial patterns and directional spatial associations between six medical resources across Wuhan city, and evaluates the impact of study area spatial form (considered as a new dimension of spatial scale) on spatial analysis. The unique morphology of Wuhan city, which is bisected by the Yangtze River, is used to assess the impacts on LCLQ analysis and the seeking behaviour of medical resources.

Results: This paper demonstrated the impacts of the spatial form of a study area on the global and local values of LCLQ of local-level medical resources. When splitting the city into multiple data sets (e.g. regions A and B in this paper), the global and local LCLQ values for pharmacies, clinics and community hospitals changed signficantly in both regions after the spatial partition. The border areas between regions $\mathrm{A}$ and $\mathrm{B}$ were influenced most.

Conclusion: This paper focused on the impacts of the unique spatial form of the study area created by large-scale natural barriers. we should not ignore the impacts of the unique spatial form of the study area created by large-scale natural barriers such as mountains, rivers or lakes. The findings highlight another form of multiscale analysis in urban GIS.
\end{abstract}

Keywords: Medical resource distribution; health GIS; localised colocation quotient; study area spatial form; multi-scale, Wuhan

\section{Introduction}

Since the introduction of economic reform and the open door policy in 1978, China began a programme of rapid urbanisation, experiencing the fastest rate of economic growth in the world, but at the cost of significant environmental degradation. The growth of its ageing population, with increased health burdens [1], has created new challenges for urban sustainability. In 2019 a fifth of the population (1.94m people) in Wuhan municipality were aged 60 and above. Of this, $2.9 \%$ (261,200 people) were senior citizens aged $\geq 80$, an increase of 10,300 since 2018 [2]. To meet the needs of the growing ageing population, the municipal government is investing heavily in medical resources, but in order to address the spatial inequality in residents' accessibility to these medical resources, additional quantitative evidence on their spatial patterns is needed [3, 4]. As such, policy makers are concerned with the following questions: what are the key characteristics of the spatial 
distribution of medical resources? What spatial associations exist between different categories of medical resources? Do spatial associations differ on each side of the Yangtze River? The availability of point of interest data, which can be freely captured from the internet using data crawling techniques [5-7], means the methods presented in this study can be easily replicated in other cities.

In the current literature, the majority of studies on medical resources have focused on analysing the spatial relationships between medical resources and community residents, such as the spatial distribution characteristics of various health resources in cities [8], the inequality of resource distribution [9], the spatial configuration of medical resources at different levels in different regions [10], and the supply mode and accessibility to medical resources [11]. Such studies frequently use regression models, such as ordinary least squares regression (OLS) [12], geographically weighted regression (GWR) [13, 14], and logistic regression (LR) [15] to analyse spatial patterns. The majority of the studies used aggregate data from national censuses, targeting urban spatial units at a range of scales from sub-district to community $[16,17]$. Geographically weighted analytical methods, including regression and principal component analysis, have been proven effective and efficient in dealing with spatial heterogeneity, specifically spatial non-stationarity. However, GIS applications for these methods, commonly used for urban governance or management, do not analyse individual spatial interactions. Moreover, aggregating data at a particular scale or spatial unit leads to the modifiable areal unit problem (MAUP) [18-20]. For example, changing the spatial extent of the study area in a GIS analysis can create multi-scale effects, which have been highlighted in many analytical methods, such as GWR [21], logistic regression [22], spatial autocorrelation analysis [23, 24], cluster analysis [25], and spatial differentiation [26].

In recent years, the growth of big data technology has increased the availability of point data. Point of interest (POI) data have been increasingly used for urban analytics because of their low cost and high temporal resolution [27-31]. Many POI data take the form of categorical rather than interval and ratio variables, and require specific analytical methods suitable for measuring spatial associations and heterogeneity of categorical point data. In contrast to other methods for measuring spatial association (such as Moran's I for interval and ratio data), the co-location quotient between multiple categories is particularly suitable for urban analytics. Leslie \& Kronenfeld (2011) developed a global colocation quotient (GCLQ) and subsequently Cromley et al. (2014) developed a local colocation quotient (LCLQ) by incorporating a geographical weighted method into the global colocation quotient [32]. Further advances included Wang et al.'s (2017) application of a Monte Carlo simulation to create a test of the statistical significance of the co-location quotient results. Co-location quotients are now widely used in urban studies [33]. Studies to date have focused on determining the the nature of spatial associations between categorial variables, such as the colocation between trees and business establishments [34], the spatial co-occurrences of food sources (convenience stores, fast food outlets) and pharmacies [35], the spatial correlation between transportation infrastructure and the location of competing retail firms [36], the spatial relationship between types of crime and facility such as bars, schools, shops [37], the spatial association between types of crimes and types of land use [33], the spatial correlation patterns between intersections and crashes (pedestrian- or cyclist-involved) [38], associations between fires and land-use types [39], crashes of various traffic accident (minor injury, major injury, and fatal) [40], and the spatial associations between cycling activities and urban facilities [41].

However, there are still some shortcomings in the published literature. To date, no studies have used the co-location quotient to measure the spatial heterogeneity or directional spatial association between types of medical resources. Nor have any studies explored the multi-scale effect on co-location quotient analysis. Lastly, the impact of the spatial form of the study area on the scale effect, including the influence of natural barriers such as mountains, rivers or lakes, has not been evaluated. This paper explores all these issues, applying both global and local co-location quotients to medical resource POI data from Wuhan City. 
This paper is organised as follows. Section 2 describes the study area and data sources, then explains the global and local (geographically weighted) colocation quotient methods used. Section 3 presents and interprets the analytical results. This is followed by a discussion of multi-scale effects in section 4 and conclusions in section 5 .

\section{Materials and Methods}

\subsection{Study Area}

Wuhan, the capital of Hubei Province, is located in central China between $113^{\circ} 41^{\prime}-115^{\circ} 05^{\prime}$ E, $29^{\circ} 58$ ' $-31^{\circ} 22^{\prime} \mathrm{N}$ [42]. The highest concentration of urban socio-economic activites and residential buildings is located in the seven urban districts in central Wuhan (Figure 1). In 2020, this area $(863 \mathrm{~km} 2)$ had a population of 11.2 million people and a GDP of 1.62 trillion RMB.

In order to follow the national 'Healthy China 2030' strategy [43], Wuhan municipality developed a range of policies, such as the "Healthy Wuhan 2035 Plan" in 2018 and "Wuhan People's Government's Implementation Opinions on Accelerating the Development of the Health Service Industry", with the aim of improving the health and quality of life of its population [44].

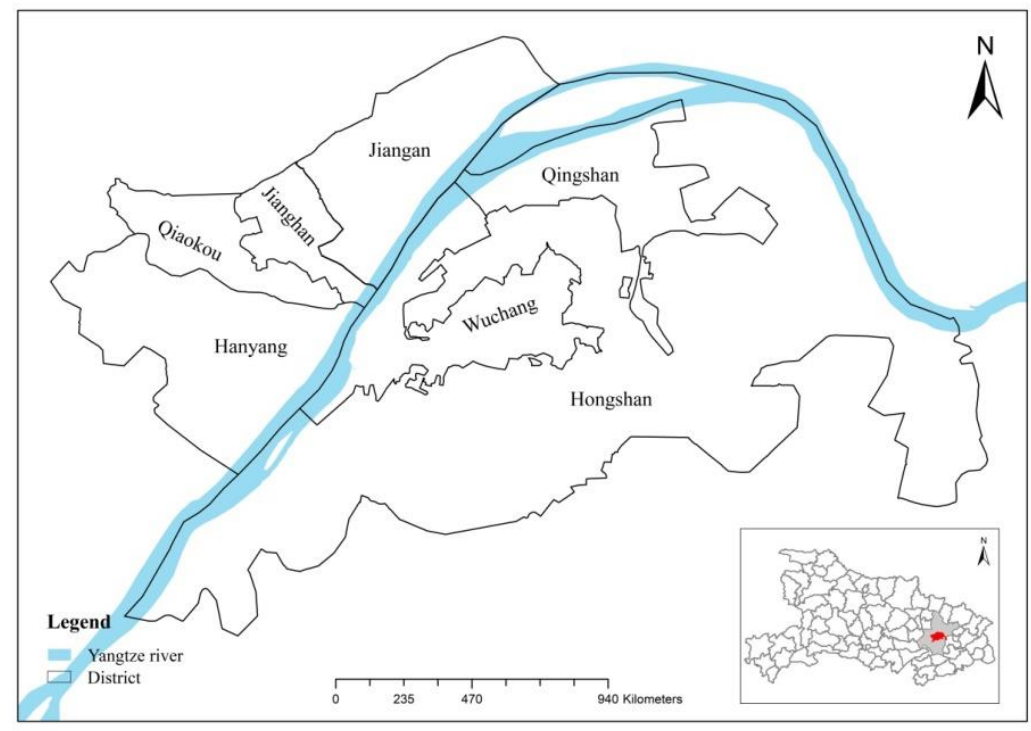

Figure 1. The location of the study area in central Wuhan, Hubei province

\subsection{Data sources}

In 2015, the General Office of the State Council issued "Guidance on Promoting the Construction of a Hierarchical Medical System", which advocated, "primary treatment at the grass-roots level, two-way referrals, separation of emergency and non-urgent treatments"[45].

Medical services in urban China are generally divided into two systems [46]: basic services, including pharmacies, clinics, and community hospitals; and professional services including specialized, general and $3 \mathrm{~A}$ hospitals ${ }^{1}$ (Figure 2).

\footnotetext{
13A hospitals are the highest level in China's classification of medical resources.
} 


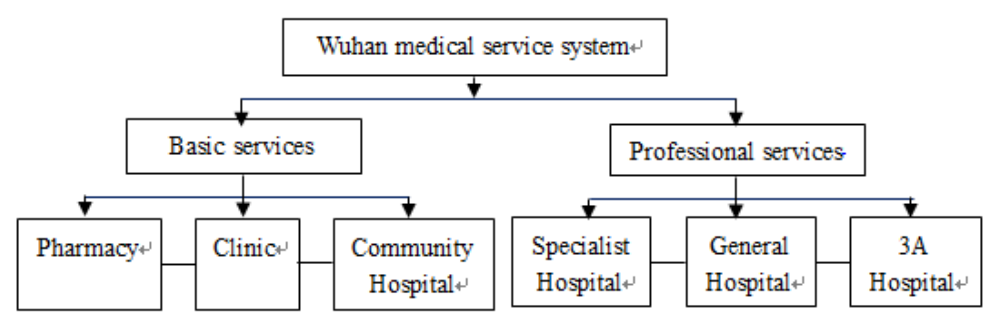

Figure 2. The hierarchy of Wuhan medical service system

In this study medical resources refers to the medical services shown in Figure 2, which includes the personnel, buildings, beds, facilities and equipment, budget, knowledge, skills and information required to operate them [47]. Hospitals were allocated to the basic or professional services group based on service demand, scale and quality [48, 49]. Community hospitals typically provide basic disease prevention, basic treatments, and nursing whereas specialist, general and $3 \mathrm{~A}$ hospitals provide medical services for complex or severe diseases.

The location and classification of all medical resources within the study area were captured from Amap API (https://developer.amap.com/product/map) in December 2019 using a web crawling technique. Preliminary data processing included coordinate correction, a coordinate point reverse check, and address information correction and completion. 5,401 POI data were collected in total (Table 1). Each POI included the medical resource name, identity number, latitude and longitude coordinates, and specific address. District boundary and road network data were also collected from the National Basic Geographic Information System database.

Table 1. Classification of medical resources in the study area

\begin{tabular}{cccc}
\hline ID & Classification & Category & Count \\
\hline 1 & & Pharmacy & 2383 \\
2 & Basic & Clinic & 1548 \\
3 & & Community Hospital & 391 \\
4 & & Specialist Hospital & 737 \\
5 & Professional & General Hospital & 297 \\
6 & & 3A Hospital & 45 \\
\hline & Total: & & 5401 \\
\hline
\end{tabular}

\subsection{Methods}

\subsubsection{Global Colocation Quotient}

To analyse the colocation patterns of various point sets, Leslie \& Kronenfeld (2011) proposed the global CLQ which measures the overall extent to which category A points (e.g. one type of medical resource) are dependent on category B points (e.g. another type of medical resource) [33]. In contrast to other methods such as Moran's I, global CLQ uses nearest neighbours rather than Euclidean distance to quantify the spatial association between different sample populations. Where a point has multiple nearest neighbours within a bandwidth each nearest neighbour is assigned an equivalent weight as shown in Equation 1.

$$
N_{A \rightarrow B}=\sum_{i=1}^{N_{A}} \sum_{j=1}^{n n_{i}} \frac{f_{i j}}{n n_{i}}
$$

Where $i$ is category A, $\mathrm{nn}_{\mathrm{i}}$ denotes the $i$ th nearest neighbour, $j$ is the number of nearest neighbours to point $i . f_{i j}$ is a binary variable, indicating whether point A's nearest neighbour $j$ is type B ( 1 means yes, 0 no). $N_{A \rightarrow B}$ denotes the number of type A points that have type B points as their nearest neighbours. The global CLQ is calculated using Equation 2. 


$$
G C L Q_{A \rightarrow B}=\frac{N_{A \rightarrow B} / N_{A}}{N_{B} /(N-1)}
$$

Where $N_{A}$ and $N_{B}$ represent the number of type A and B medical resources respectively, $N_{A \rightarrow B}$ is the number of type A points (Equation 2), whose nearest resource is type B. $\mathrm{N}$ is the total number of medical resources (5401) in the study area (Table 1). When calculating expectations, the denominator is $(\mathrm{N}-1)$ instead of $\mathrm{N}$, because a point cannot be its own neighbour [37].

$\mathrm{GCLQ}_{A \rightarrow B}$ measures the extent to which type A points are attracted to type B points [34]. When $\mathrm{GCLQ}_{A \rightarrow B}=1$, type A and B points are both randomly distributed within the study area. That is, the proportion of type $B$ nearest neighbours to type A points is equal to the overall proportion of type B points in the sample. When $\mathrm{GCLQ}_{A \rightarrow B}>1$, it means that category A is dependent on $\mathrm{B}$, and the number of the nearest neighbours of type $\mathrm{B}$ is higher than expected. The greater the value, the more dependent it is. When $\mathrm{GCLQ}_{A \rightarrow B}<1$, A and B are mutually exclusive, that is, the number of type $B$ nearest neighbours is lower than expected. The smaller the value, the greater the spatial independence between the two types. The smallest possible value of GCLQ ${ }_{A \rightarrow B}$ is zero, which indicates there are no type $B$ nearest neighbours to type A points. The spatial association expressed by $\mathrm{GCLQ}_{A \rightarrow B}$ is unidirectional, because A and B may have an asymmetric nearest neighbour dependency. If A's nearest neighbour is $\mathrm{B}$, and B's nearest neighbour is not A, then $\mathrm{GCLQ}_{A \rightarrow B}>\mathrm{GCLQ}_{B \rightarrow A}$ which indicates that the spatial attraction of $\mathrm{B}$ to $\mathrm{A}$ is greater than that of $\mathrm{A}$ to $\mathrm{B}$.

\subsubsection{Local Colocation Quotient}

The local colocation quotient (LCLQ) developed by [32] was used to analyse the spatial heterogeneity of medical resources and map clusters of points with high spatial associations. Compared with the global colocation quotient, the LCLQ produces maps that are easier to interpret [37]. LCLQ is calculated as follows in Equation 3:

$$
L C L Q_{A_{i} \rightarrow B}=\frac{N_{A_{i} \rightarrow B}}{N_{B}(N-1)}
$$

WhereN and $\mathrm{N}_{B}$ are the same as in equation (2), $N_{A_{i} \rightarrow B}$ represents the geographically weighted average of type B points within the bandwidth of type A points, as shown in Equation 4.

$$
\begin{gathered}
N_{A_{i} \rightarrow B}=\sum_{j=1(j \neq i)}^{N}\left(w_{i j} f_{i j} / \sum_{j=1(j \neq i)}^{N} w_{i j}\right) \\
w_{i j}=\exp \left(-0.5 * \frac{d_{i j}^{2}}{d_{i b}^{2}}\right)
\end{gathered}
$$

$A_{i}$ represents the i-th type A point, $f_{i j}$ indicates whether the nearest neighbour to point $A_{i}$ is point $\mathrm{B}$, then $f_{i j}=1$, or if not $f_{i j}=0, w_{i j}$ is the weight of point $\mathrm{j}$, indicating the importance of individual $j$ to individual $A_{i} . d_{i j}$ represents the distance between point $A_{i}$ and point $j, d_{i b}$ represents the bandwidth of the search area for neighbours of $A_{i}$. The Gaussian kernel density functionin equation (5) was used to assign the geographical weight value to each neighbour of $A_{i}$. The farther each neighbour is from $A_{i}$, the smaller its weight will be. An adaptive bandwidth is typically used for study areas with heterogeneous density e.g. one containing both urban and rural areas. A bandwidth determined by distance rank guarantees that each type A point has exactly the same number of neighbours, making the results more robust and reliable [37]. The local location quotient (LCLQ) is expected to be 1 . Therefore, if $\operatorname{LCLQ}_{A_{i} \rightarrow B}$ is greater than 1, it indicates that $A_{i}$ is nearest to a type $\mathrm{B}$ point. The greater the value, the stronger the association between types. Conversely, if $\operatorname{LCLQ}_{A_{i} \rightarrow B}$ is less than $1, A_{i}$ does not have a type $\mathrm{B}$ point as its nearest neighbour. A Monte Carlo simulation was repeated 1000 times to test the statistical significance of the local location quotient results [37]. 


\section{Results}

This section may be divided by subheadings. It should provide a concise and precise description of the experimental results, their interpretation, as well as the experimental conclusions that can be drawn.

\subsection{Spatial pattern of medical resources}

The kernel density analysis indicated the high concentration and clustering of all types of medical resources across the city centre, bisected by the Yangtze River (Figure 3).
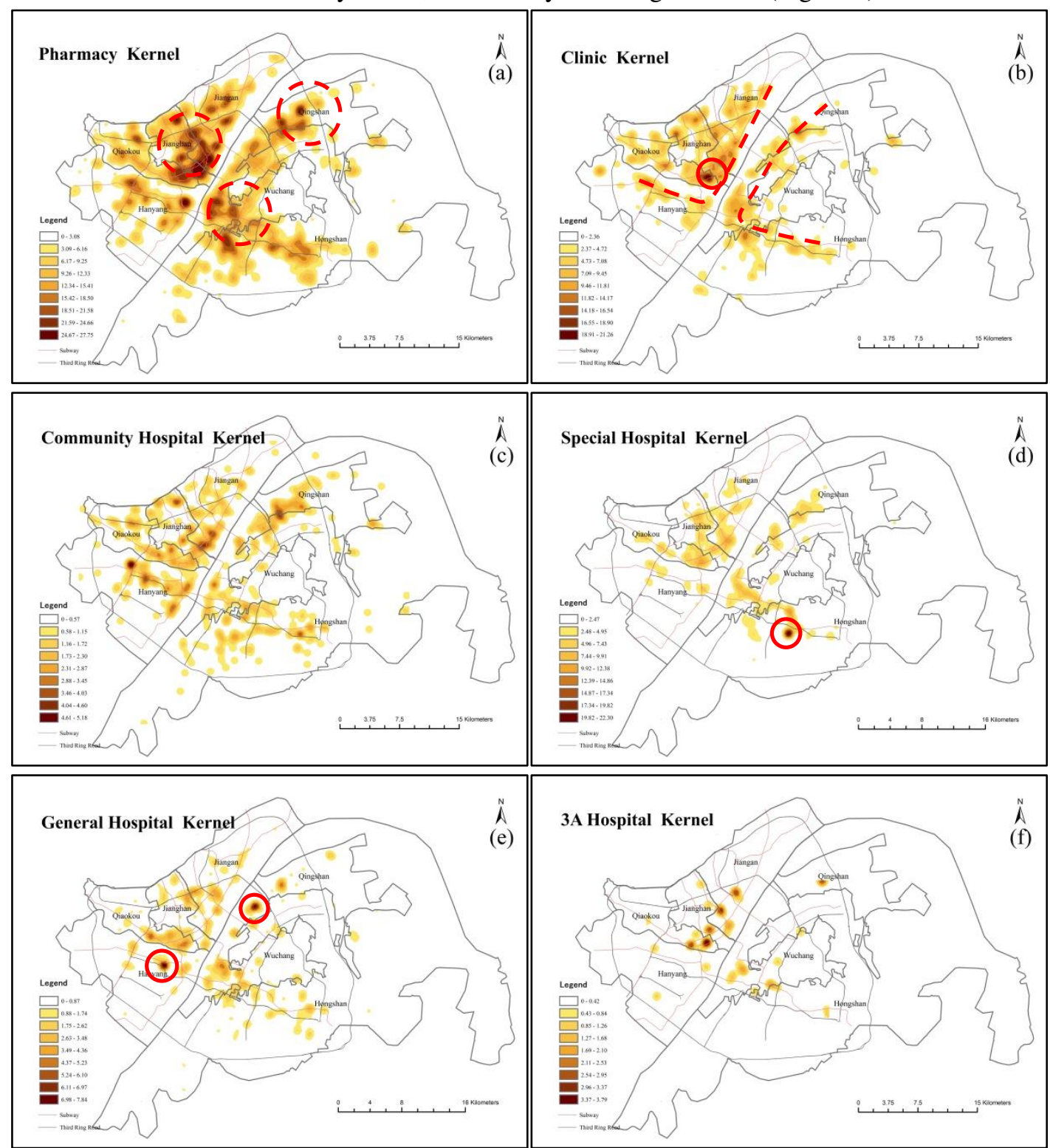

Figure 3. Kernel density estimation of (a) Pharmacies; (b) Clinics; (c) Community Hospitals; (d) Special Hospitals; (e) General Hospitals; and (f) 3A Hospitals.

Pharmacies were relatively widely distributed within the study area, with three high-density clusters represented by the red circles in Figure 3a. Clinics were distributed along the banks of the Yangtze River, with an area of high density in the Qiaokou District (Figure 3b). The distribution of community hospitals followed a similar pattern although the areas of highest density were located in Qingshan District, Hanyang District, Jiangan District and other former urban areas (Figure 3c). Community hospitals were the most evenly distributed of all medical resources across 
the city centre and suburban areas. This reflects the boundaries of the smallest administrative unit to which medical resources are allocated in China's medical system [50, 51].

The distribution of special hospitals was relatively scattered. With the exception of Hongshan district, most districts were characterised by a scattered distribution of special hospitals following the subway line. It also suggests that accessibility is a key factor in siting special hospitals (Figure 3d). The high-density areas of general hospitals were concentrated in Wuchang and Hankou districts, presenting a "dual-core" pattern either side of the Yangtze River (Figure 3e). 3A hospitals were mainly concentrated in the three former districts of Jiangan, Jianghan and Qiaokou (Figure 3f). Newer districts only had branches or subunits of these hospitals, such as Tongji Hospital Guanggu branch and Union Hospital Jinyinhu branch.

These spatial patterns suggests a degree of inequality, where high-level medical resources are excessively concentrated in central urban areas. Due to the influence of historical development of Wuhan, the old urban area still has the highest concentration of high-level medical resources. It also shows the lack of high-level medical resources in the new urban areas around the city centre. This may reflect the sparse residential areas and low population density in the new urban areas, which leads to insufficient provision and allocation of high-level medical resources.

The results also show the great disparity in the spatial distribution of various medical resources between two sides of the river. By calculating the number of medical resources per capita per unit area of each district (Figure 4), it was found that pharmacies were mainly distributed in the Jianghan District, special hospitals were mainly distributed in the Qingshan district, and clinics were mainly distributed in the Qiaokou district. There was a relatively small spatial disparity in the distribution of community hospitals, general hospitals and 3A hospitals. This may reflect the fact that the site selection, investment and construction of these resources are determined by central government $[49,52,53]$, while pharmacies, clinics and specialist hospitals are mostly privately owned. To improve the equality and equity of public medical resource distribution, the government should distribute medical resources in accordance with the current population distribution and demographic structure across the city. There are obvious differences in the form, pattern and quantity of pharmacies, clinics and special hospitals which suggests the coordination of different resources in Wuhan is still inadequate.

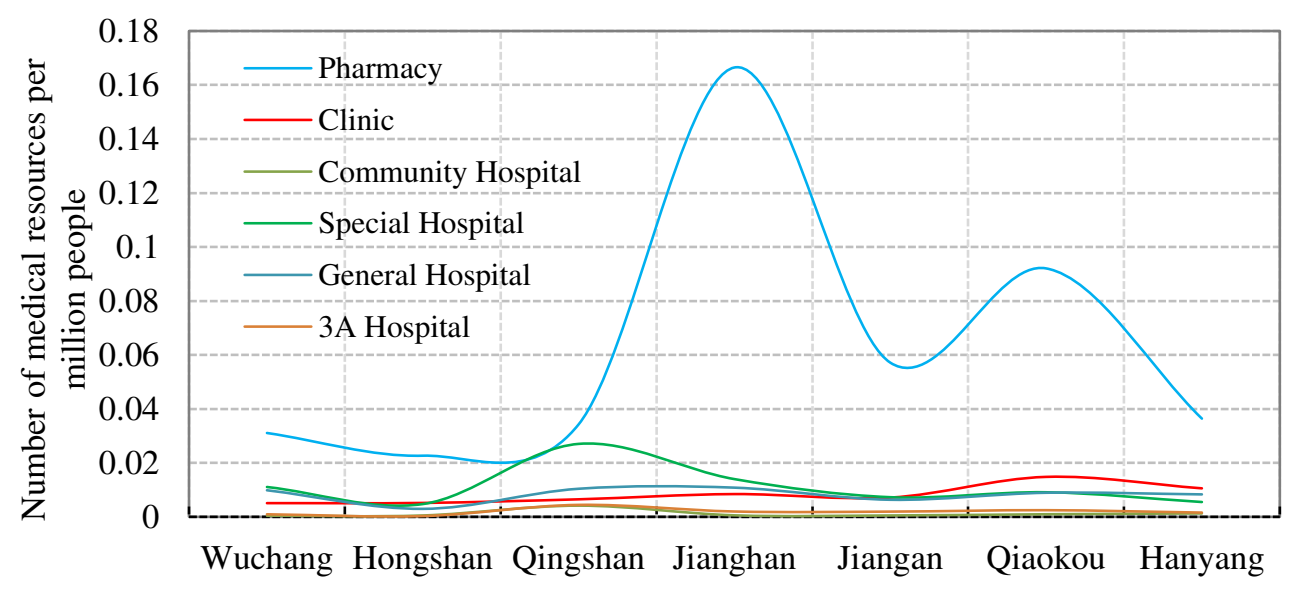

Figure 4. Distribution of medical resources between the seven urban districts in Wuhan.

\subsection{Spatial associations}

3.2.1 Global location quotient analysis (GCLQ)

Table 2. Global colocation quotient for all medical resources.

\begin{tabular}{ccccccc}
\hline Wuhan & Pharmacy & Clinic & $\begin{array}{c}\text { Community } \\
\text { Hospital }\end{array}$ & $\begin{array}{c}\text { Special } \\
\text { Hospital }\end{array}$ & General Hospital & $\begin{array}{c}\text { 3A } \\
\text { Hospital }\end{array}$ \\
\hline
\end{tabular}




\begin{tabular}{|c|c|c|c|c|c|c|}
\hline Pharmacy & $\begin{array}{c}2(1.175) \\
* * *\end{array}$ & $\begin{array}{c}2(1.077) \\
* *\end{array}$ & $\begin{array}{c}1(1.228) \\
* *\end{array}$ & 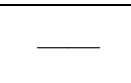 & $\begin{array}{c}24(0.934) \\
* *\end{array}$ & $\begin{array}{c}11(0.744) \\
* * *\end{array}$ \\
\hline Clinic & $\begin{array}{c}3(1.074) \\
* * *\end{array}$ & $\begin{array}{c}1(1.657) \\
* * *\end{array}$ & - & $\begin{array}{c}5(0.909) \\
* *\end{array}$ & $25(0.854)^{* * *}$ & $\begin{array}{c}11(0.665) \\
* * *\end{array}$ \\
\hline $\begin{array}{c}\text { Community } \\
\text { Hospital }\end{array}$ & $\begin{array}{c}4(1.186) \\
* * *\end{array}$ & $\begin{array}{c}10(1.077) \\
*\end{array}$ & & - & $\begin{array}{c}1(2.279) \\
* * *\end{array}$ & $\begin{array}{c}5(0.093) \\
* * *\end{array}$ \\
\hline Special & $20(0.97)$ & $2(0.835)$ & $7(0.869)$ & $1(2.654)$ & $1(1.525)$ & $2(2.197)$ \\
\hline Hospital & $*$ & $* * *$ & $* * *$ & $* * *$ & $* * *$ & $* * *$ \\
\hline General & $8(0.902)$ & $25(0.844)$ & $1(2.173)$ & $1(1.646)$ & $1(6.159)$ & $1(4.874)$ \\
\hline Hospital & $* * *$ & $* * *$ & $* * *$ & $* * *$ & $* * *$ & $* * *$ \\
\hline 3A Hospital & $\begin{array}{c}25(0.842) \\
* * *\end{array}$ & $\begin{array}{c}24(0.711) \\
* * *\end{array}$ & $\begin{array}{c}25(0.591) \\
* * *\end{array}$ & $\begin{array}{c}4(2.001) \\
* * *\end{array}$ & $\begin{array}{c}5(3.757) \\
* * *\end{array}$ & $\begin{array}{c}1(29.813) \\
* * *\end{array}$ \\
\hline
\end{tabular}

Note: Taking "2(1.175)***" as an example, " 2 " is the bandwidth size, " 1.175 " is the GCLQ value, and "***" indicates significance at $1 \%$ level, “**” indicates significance at $5 \%$ level,. Highlighted areas indicate the GCLQ values greater than 1.

The GCLQ results show significant 'self proximity' within each type of medical resource except community hospitals (Table 2). That is, pharmacies, clinics, special hospitals, general hospitals and 3A hospitals were not randomly distributed, but showed a degree of spatial clustering. Varying degrees of spatial association were evident within each of the five resource categories, ordered as follows: 3A hospitals (29.813), general hospitals (6.159), special hospitals (2.654), clinics (1.657), and pharmacies (1.175). 3A hospital showed the highest level of spatial clustering, followed by general hospitals. It is interesting to see that the higher the level of medical resource, the higher the level of spatial association. Higher-level medical resources are more commerically driven so spatial agglomeration might be a more important consideration than for lower level resources which are provided by local government, with the aim of equal and even allocation at the urban community level.

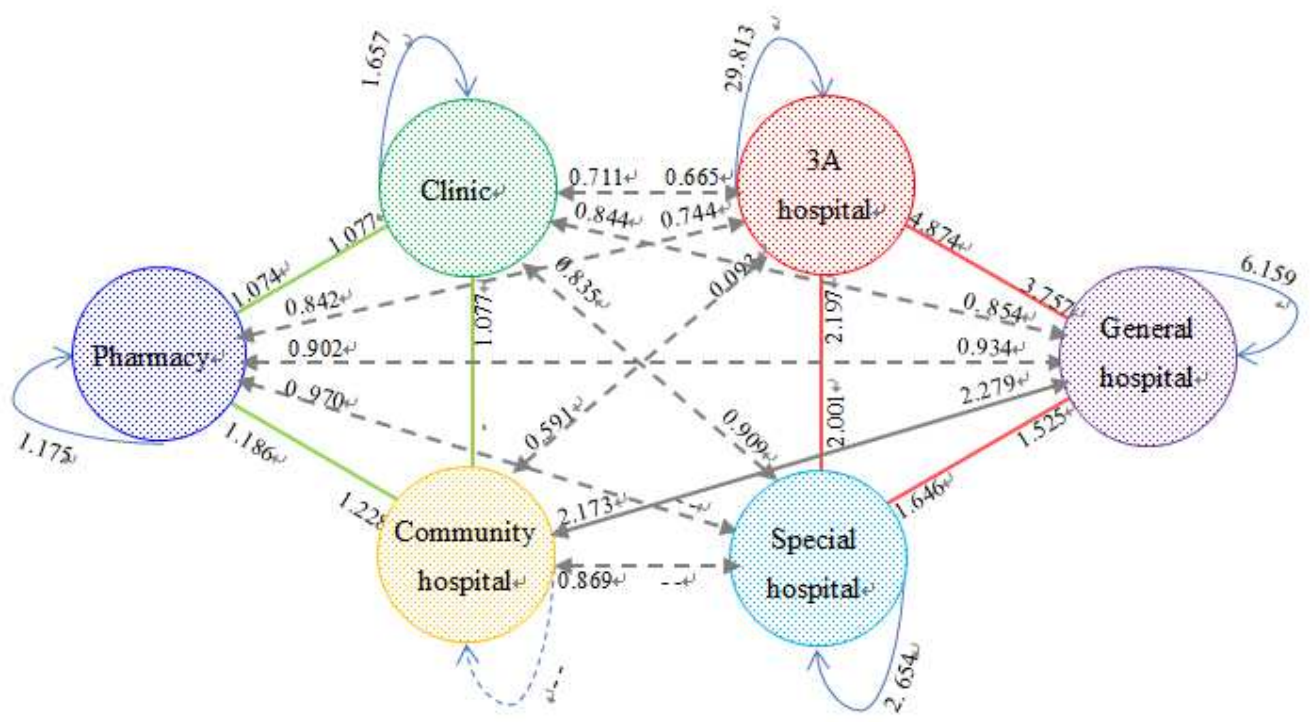

Figure 5. Directional spatial association between medical resource categories.

The GCLQ results (Table 2) also showed a degree of symmetric association - two-way co-location - between medical resource types. The GCLQ value was greater than 1 between each type of medical resource in the professional category (special hospitals, general hospitals and 3A hospitals) (Figure 5). The strongest co-location was between 3A and general hospitals (Figure 5). This suggests these resources complement each other in terms of service scale and target diseases $[54,55]$. Two other pairings were evident: pharmacies and clinics, and pharmacies and community hospitals both exhibited some symmetric association, although the degree of two-way co-location 
was less than that within the professional category (Figure 5). The significance of pharmacies in the spatial associations detected is explained by their central role in local communties, providing longer opening hours than hospitals, and easy access to medication [56, 57], particularly after a diagnosis from a clinic or community hospital visit. There was an asymmetric association between clinics and community hospitals, the former being dependent on the latter (GCLQ $=1.077$ ) but the opposite not being significant. This is because there is only one community hospital per community so is likely to be located in an area with good accessibility to all residents. However clinics, being mostly private, smaller scale services, are typically located near residential areas. As such, community hospitals do not have a significant spatial association with clinics.

Only one two-way co-location was detected between a pair of resources from the basic and professional categories. Community hospitals and general hospitals were spatially dependent, indicated by the community $\rightarrow$ general GCLQ value of 2.279 and a general $\rightarrow$ community GLCQ value of 2.173. These relatively high values indicate the proximal interaction between community-level and city-level (general) hospitals. Both community hospitals and general hospitals are public institutions administered by local governments, and their spatial associations is taken into consideration by planners. In addition, China's current resident medical insurance system (including the basic medical insurance system for urban workers, the medical insurance system for urban residents, and the new rural cooperative medical care system) requires residents to seek medical treatment in designated hospitals. Both community and general hospitals dominate the designated hospital list for most residents [53, 58]. This will affect the behaviour of residents and the location strategy for medical resources at this level.

Finally, there was a weak spatial association between all pairs of resources across the two categories (except community and general hospitals), as shown by GCLQ values less than 1 . This might reflect the disparate locations of city and community level services.

\subsubsection{Local colocation quotient}

The global colocation quotient (GCLQ) measures the direction and intensity of global spatial associations between medical resources within and between categories across the study area, but does not consider spatial heterogeneity (non-stationary co-location). The power of the LCLQ is its ability to map the direction and intensity of local spatial associations, i.e. co-location within a local neighbourhood.

The geographically weighted (local) co-location quotient results for all seven types of medical resources are shown in Figure 6. Only points with a $P$ value of less than 0.05 and an LCLQ value greater than 1 were mapped (Figure 6). There was a very strong spatial association between pharmacies, as indicated by the prevalence of red and orange points (Figure 6a). It is worth noting although there is a certain degree of competition between pharmacies in terms of service objectives, the pattern was dominated by a spatial agglomeration effect. This finding provides important empirical evidence of the spatial layout of pharmacies. Figure 6b shows that although there is also a significant spatial association between clinics, the spatial agglomeration was not significant. There were 392 community hospitals in the central urban area of Wuhan, only two of which showed a local spatial association, indicating weak spatial association between community hospitals. The main reason is that community hospitals are public medical resources and their spatial distribution is strictly controlled by the government. The spatial association among special hospitals was strong, with LCLQ values of greater than 4.5 for most of them. The intensity of spatial associations between general hospitals was lower than that of special hospitals, and the areas of spatial clustering were mainly distributed in the old town areas of Hankou and Hanyang. 


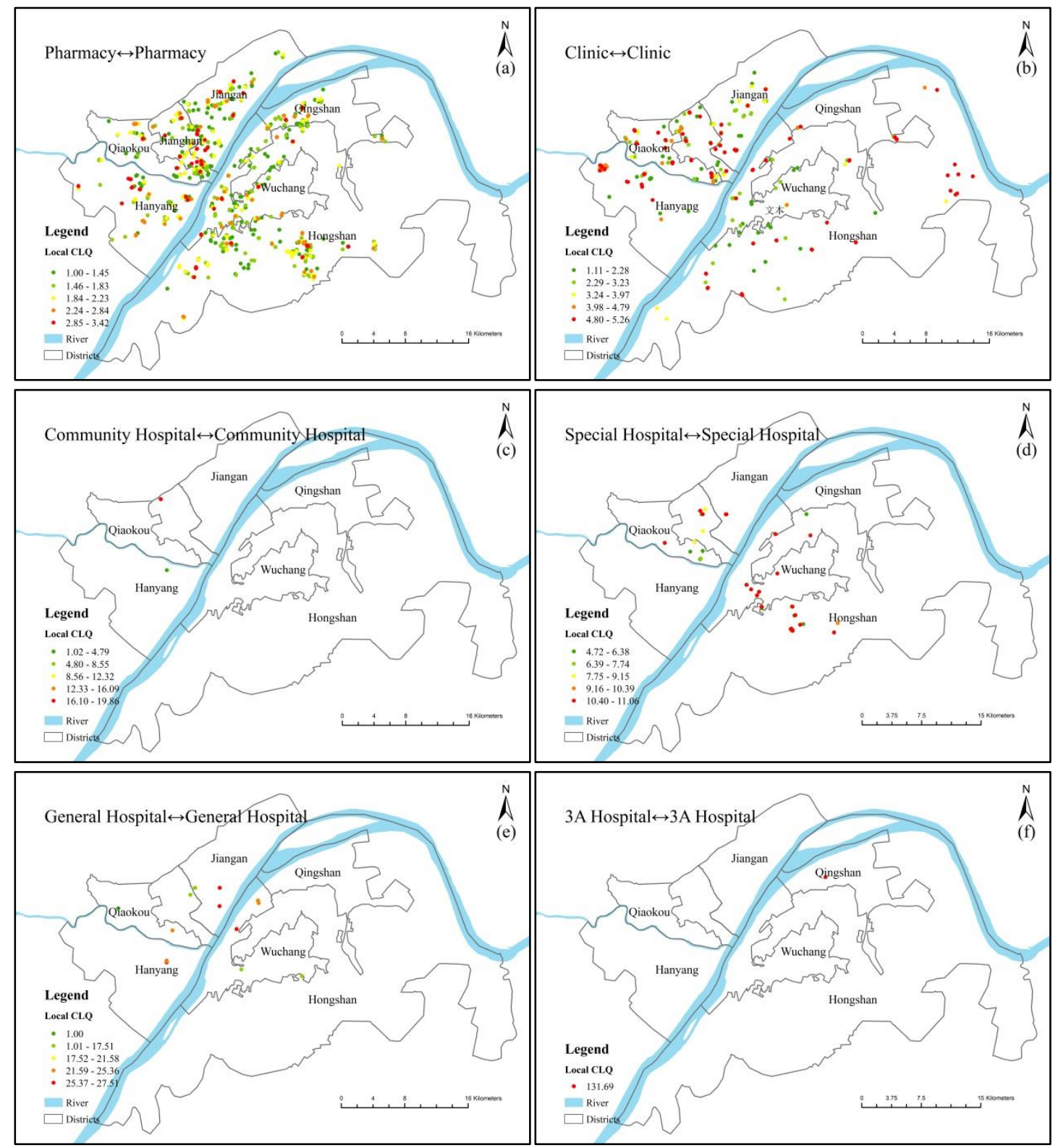

Figure 6. Local Colocation Quotient values between the six medical resources

The local co-location quotient results for basic medical resources (pharmacies, clinics and community hospitals) are shown in Figure 7. Only points with a LCLQ value greater than 1 and a $P$ value less than 0.05 were included.

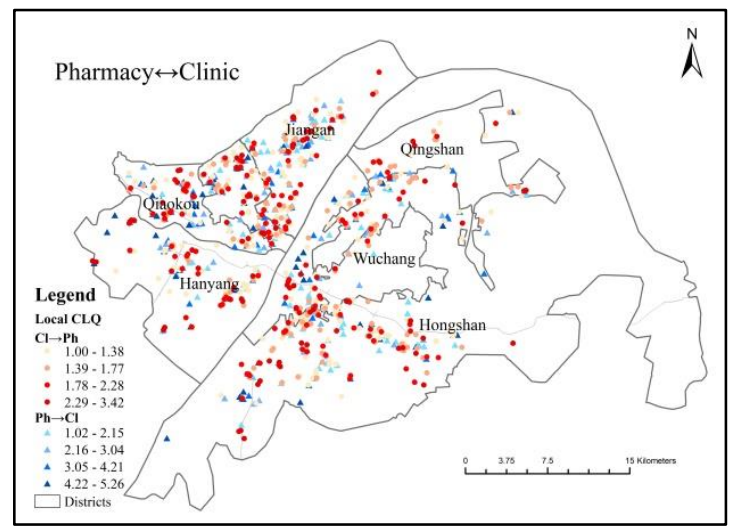




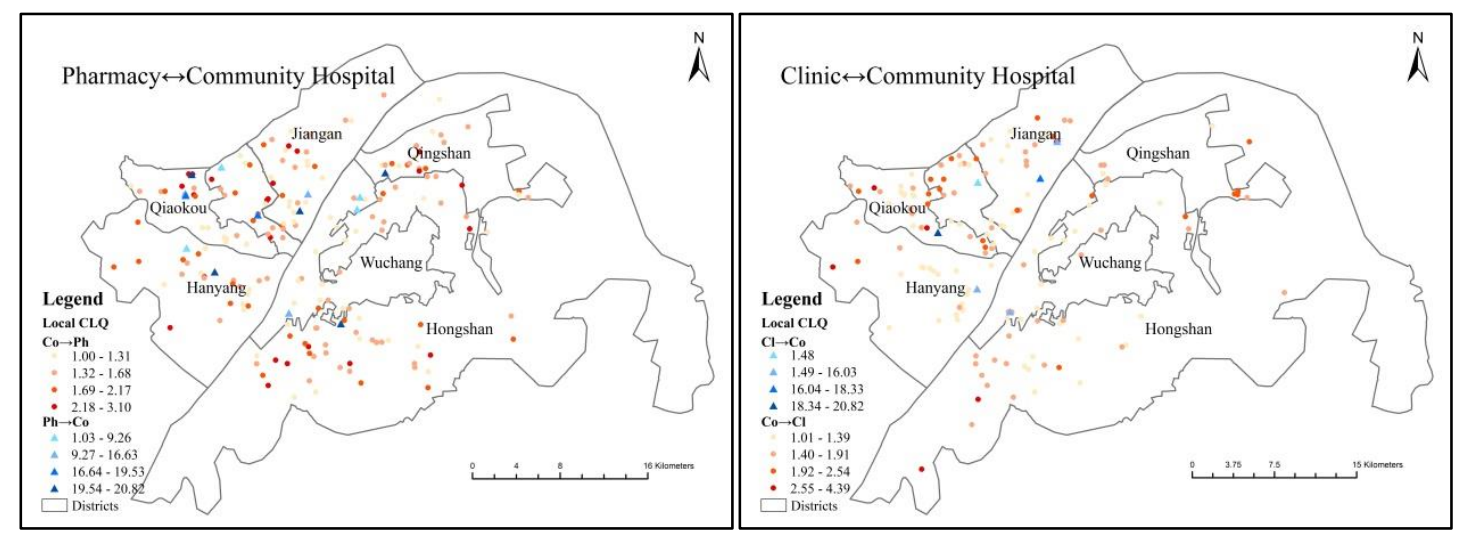

Figure 7. Spatial distribution of local colocation quotient values between the three types of basic medical resources (clinics, pharmacies and community hospitals)

The results showed that pharmacies and clinics were significantly more interdependent than other medical resources. Community hospitals depended on pharmacies, but only by a small degree, as indicated by the LCLQ values of between 1 and 3.42. Pharmacies are well distributed around the community to sell over-the-counter drugs, whereas community hospitals mainly provide medical services for designated communities. The construction and location of community hospitals are decided by government planners based on accessibility and equity of residents' medical treatment. Therefore, the spatial dependence of community hospitals on pharmacies is small. In addition, the number of pharmacies that have a spatail dependence on community hospitals is small, but the degree of dependence is large, with LCLQ values between 1 and 20.82. This is because the majority of pharmacies serving the community residents are widely distributed around the community. A small number of pharmacies are dependent on community hospitals as they provide professional auxiliary medical services for community hospitals, or are recommended by community hospitals. Therefore, these pharmacies have high-degree association with community hospitals. Thirdly, community hospitals generally rely on clinics. However, due to the strong competitive relationship between clinics and community hospitals, and the fact that clinics are smaller than community hospitals in terms of facilities and scale, most clinics do not rely on community hospitals.

The LCLQ values for professional medical resources are shown in Figure 8, which includes only points with a $\mathrm{P}$ value of less than 0.05 :

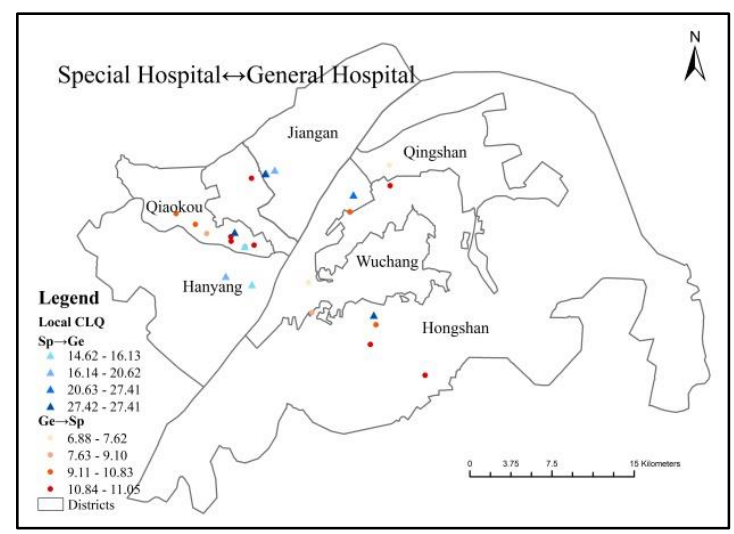




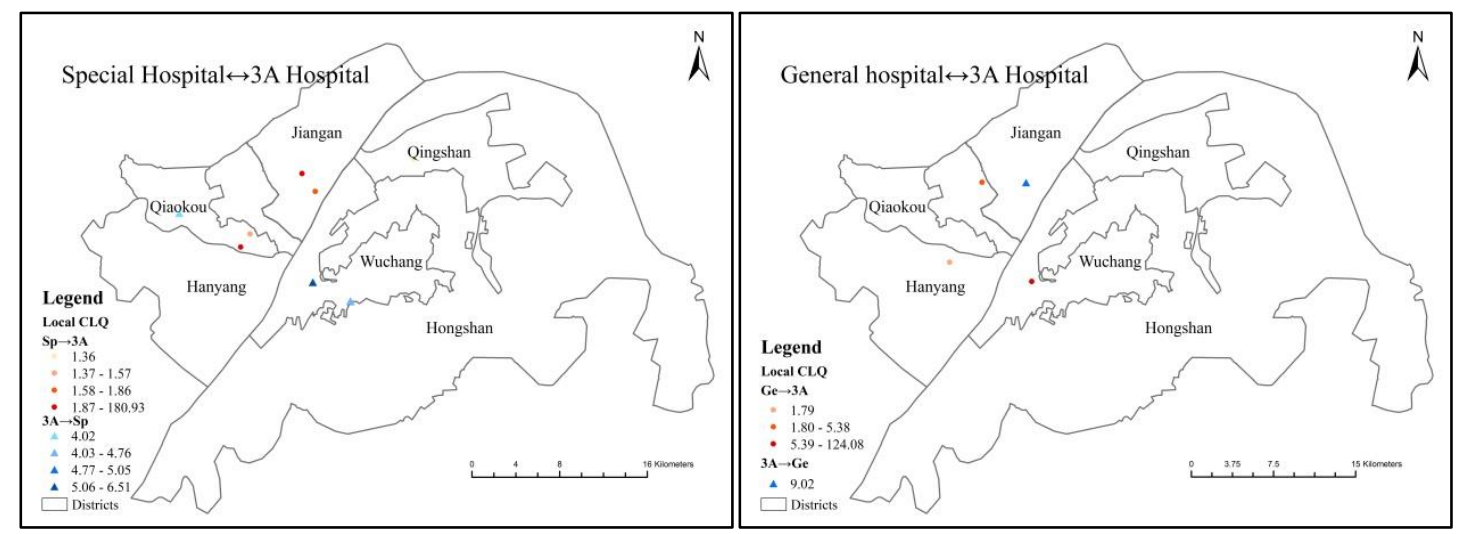

Figure 8. Spatial distribution of local colocation quotient values between General Hospitals, Special Hospitals and 3A Hospitals

Special hospitals had a strong spatial association with general hospitals. The LCLQ values ranged from 14.62 to 27.42 . The spatial association of special hospitals to $3 \mathrm{~A}$ hospitals was mainly concentrated in the vicinity of Tongji and Xiehe Hospitals. Tongji Hospital and Union Hospital have a good reputation and patients from all over the country seek medical treatment here. For this reason, many special hospitals are attached to Tongji Hospital and Union Hospital, combining their specialisms to offer targeted medical services.

General hospitals had a strong spatial association with $3 \mathrm{~A}$ hospitals. One reason is that China's medical insurance system requires patients who need hospitalization to go to designated hospitals for treatment. Patients who cannot be treated in a general hospital are sent to a designated 3A hospital. Also, Wuhan Municipal Government has issued regulations that all 3A hospitals in the city provide assistance to general hospitals, and some general hospitals are affiliated to 3A hospitals [59]. Therefore, general hospitals have a strong spatial association with 3A hospitals.

3A hospitals were less dependent on the location of special hospitals and general hospitals, although this was based on a relatively small sample of 45 hospitals in the central urban area of Wuhan. In addition, 3A hospitals have more specialist equipment and doctors than special hospitals or general hospitals, and they offer different services, which has formed a distinct agglomeration and competition effect. Therefore, it is difficult for special hospitals and general hospitals to compete with them. Accordingly $3 \mathrm{~A}$ hospitals have a weak spatial association with special hospitals and general hospitals (Figure 8).

The results highlight the stronger local spatial association between basic medical resources than professional medical resources.

\section{Discussion}

Understanding the spatial pattern of medical resources is a prerequisite to achieving greater spatial equality and social equity in the allocation of medical resources. This section discusses the sensitivity of the aforementioned analytical results to bandwidth size and the spatial form of the study area. The problem of MAUP in spatial analysis is well known, and includes the impacts of the spatial division of the study area on modelling results [21].

In this paper, the global CLQ developed by Leslie et al. (2011) was used to estimate bandwidth. The bandwidth was initially set at first-order neighbours before gradually increasing the number of neighbours. The GCLQ was calculated for each increase. A Monte Carlo simulation, repeated 1000 times, was used as a significance test. Finally, the number of neighbours (bandwidth) was determined by comparing the results. Using a the same bandwidth to measure the global and local locations of all health resources can lead to biased results. To ensure robust, 
reliable results, this study used various bandwidths, each suited to the particular spatial distribution characteristics of the different medical resources.

In this study, the Yangtze River divides the central urban area of Wuhan into two parts: region A (Hanyang, Jiangan, Jianghan and Qiaokou districts) and region B (Hongshan, Wuchang and Qingshan Districts). The resulting spatial form of the study area significantly affected the public use of medical resources. It might be assumed that use of higher level $3 \mathrm{~A}$ and specialist hospitals might be not sensitive to the spatial form of the study area for three reasons. Firstly, these hospitals disagnose and treat severe diseases (such as cancer) using costly specialist equipment and highly skilled doctors that are not available at lower level hospitals. As such, these hospitals have good regional and national reputation and attract patients from surrounding cities and provinces [60]. Secondly, 3A hospitals have high threshold expenses for medical treatment. In terms of China's medical reimbursement system, patients need to visit community or general hospitals for diagnosis and treatment in the first instance. They can use 3A hospitals only if the community and general hospitals cannot provide the necessary treatment. Otherwise, the treatment must be self-funded. Thirdly, these hospitals are not frequently visited by local citizens due to the high costs and long waiting times for treatment. Therefore, spatial accessibility to these hospitals is not the key consideration, rather, visiting frequency is very much dependent on the severity of disease and specialism of required treatment. By contrast, the lower-level hospitals, particularly at community level, were relatively sensitive to the spatial form of the study area as spatial accessibility and proximity of medical resources were important considerations to patients using them. Ding et al. (2018) also found that spatial accessibility and proximity were key consdierations in the use of low-cost and frequently needed health services. In this case study, citizens in region A might not wish to access community-level medical resources in region B, and vice versa, due to the impact of the river on accessibility. Therefore, it was imperative to examine how the global and local spatial associations between categorical variables responded to a change in the spatial extent of the study area that more accurately reflected the segregated seeking behaviour.

\subsection{Impacts of changes to study area extent on GCLQ results.}

Table 3 shows the GCLQ results in regions A and B. These were compared with the results for the whole study area shown in Table 2. The majority of GCLQ values increased when the study area was split into two regions, indicating greater spatial association between medical resources, and a denser, more decentralized distribution. Following partition clinics became significantly spatially dependent on community hospitals. The intensity of spatial association between medical resources generally increased as well, except between pharmacies in Region A. The spatial association between clinics and pharmacies increased from 1.074 to 1.153 in Region A and to 1.119 in Region B. The spatial association between clinics increased from 1.657 to 1.857 and 1.700 in Regions A and B respectively. The degree of spatial dependence of community hospitals on pharmacies increased from 1.186 to 1.269 in Region A and 1.190 in Region B. The spatial dependence of community hospitals on clinics increased from 1.077 to 1.800 and 1.151 in Regions A and B respectively. Therefore, the natural barrier (Yangtze River) has a significant spatial dependence on all medical resources.

Table 3. Global colocation quotient values in Regions A and B.

\begin{tabular}{cccc}
\hline Region A & Pharmacy & Clinic & Community Hospital \\
\hline Pharmacy & $3(1.043)$ & $8(1.028)$ & $5(1.139)$ \\
& $* *$ & $* *$ & $* * *$ \\
Clinic & $5(1.153)$ & $1(1.857)$ & $21(1.083)$ \\
& $* * *$ & $* * *$ & $* *$ \\
Community & $3(1.269)$ & $22(1.800)$ & \\
Hospital & $* * *$ & $* *$ &
\end{tabular}




\begin{tabular}{cccc}
\hline Region B & Pharmacy & Clinic & Community Hospital \\
\hline Pharmacy & $1(1.319)$ & $11(1.040)$ & $1(1.407)$ \\
& $* * *$ & $* * *$ & $* * *$ \\
Clinic & $3(1.119)$ & $1(1.700)$ & - \\
Community & $4(1.190)$ & $6(1.151)$ & \\
Hospital & $* * *$ & $* * *$ & - \\
\hline
\end{tabular}

Note: * taking " $3(1.043)^{* * "}$ as an example, "3" is the bandwidth size, "1.043" is the GCLQ value, "**" signifies significance at 0.05, “***” signifies significance at 0.01

\subsection{Impacts of changes to study area extent on $L C L Q$ results}

Pharmacies are the most widely used medical resource at community level in China as there is no need for a doctor's prescription to purchase the majority of medicines [61]. Pharmacies usually open from $7 \mathrm{am}-11 \mathrm{pm}$ and are typically located within a 15 minute walking distance from residential buildings [62]. As the pharmacy category had the most data points in this case study, it was used to illustrate the impacts of changing the spatial extent of the study area on LCLQ clusters. The LCLQ values between pharmacies were re-calculated for regions A and B, then shown on the same map to aid comparison of the results (Figure 9). The number of significant points $(\mathrm{P} \leq 0.05)$ decreased when the extent of the study area was changed, but the intensity of spatial associations (LCLQ $\geq 1$ ) between pharmacies increased.
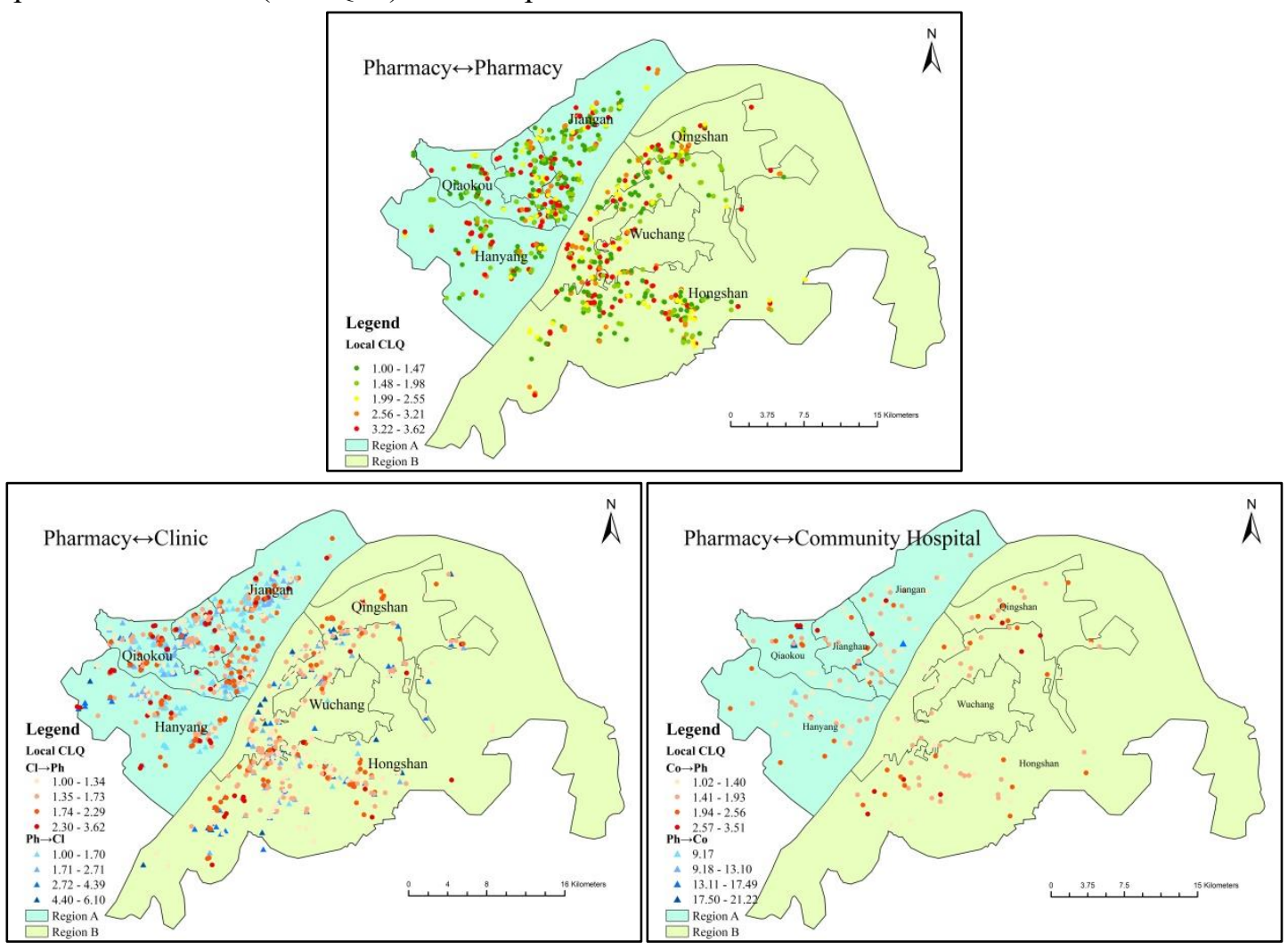

Figure 9. Local colocation quotient values in Regions A and B between basic medical resources

The change in spatial association between clinics and pharmacies was used as an example of the effect of partitioning the study area into two regions. Following partition, the LCLQ values increased significantly, and the slope of the fitted trend line ${ }^{2}$ decreased from -0.281 to -0.156 ,

2 The LCLQ value was taken as the $\mathrm{Y}$ axis and the $\mathrm{P}$ value as the $\mathrm{X}$ axis to make a scatter plot and generate a fitted trend line as follows: $\mathrm{Y}_{\mathrm{LCLQ}}^{\text {bef }}=-0.281 \mathrm{X}_{\mathrm{P}}^{\text {bef }}+0.584, \mathrm{Y}_{\mathrm{LCLQ}}^{\text {aft }}=-0.156 \mathrm{X}_{\mathrm{P}}^{\text {aft }}+0.502$. 
indicating that the local colocation quotient for pharmacies generally increased, and the $\mathrm{P}$ value generally decreased (Table 4). More specifically, 57\% of pharmacies had an increased LCLQ value, and 37\% (875) had a significantly lower P value (Table 4). 25\% (603) of pharmacies had an increased LCLQ value but a smaller P value (Table 4). It can be seen that after partitioning, LCLQ values generally increased and $\mathrm{P}$ values generally decreased. All the pharmacies were divided into four quadrants based on the LCLQ results before and after partition (Table 4). The number of pharmacies with $\mathrm{P} \leq 0.05$ and LCLQ $\geq 1$ decreased by 153 after the study area was split into two regions, reflecting the reduction in co-located pharmacies within a small scale. This indicates that pharmacies are sensitive to the spatial extent of the study area.

Table 4. Four quadrant distribution of LCLQ values relating to pharmacies before and after the study area partition.

\begin{tabular}{c|cc||c|cc}
\hline Before & LCLQ $<1$ & LCLQ $\geq 1$ & After & LCLQ $<1$ & LCLQ $\geq 1$ \\
\hline $\mathrm{P} \leq 0.05$ & 1909 & 1328 & $\mathrm{P} \leq 0.05$ & 1885 & 1175 \\
$\mathrm{P}>0.05$ & 1575 & 2337 & $\mathrm{P}>0.05$ & 1779 & 2310 \\
\hline
\end{tabular}

Note: The numbers refer to the number of pharmacies in each quadrant

Finally, the changing spatial distribution characteristics of the pharmarcy category were compared (Figure 10). Pharmacy-pharmacy LCLQ values increased significantly (Fig. 10a) after the study area was partitioned but LCLQ values significantly decreased (Fig. 10b). Figure 10c shows the pharmacies with an increased LCLQ value but smaller $P$ value. The pharmacies with significant changes were mostly distributed near the boundary between the two regions along the Yangtze River, whereas those near the centre of each region (A and B) did not change very much.
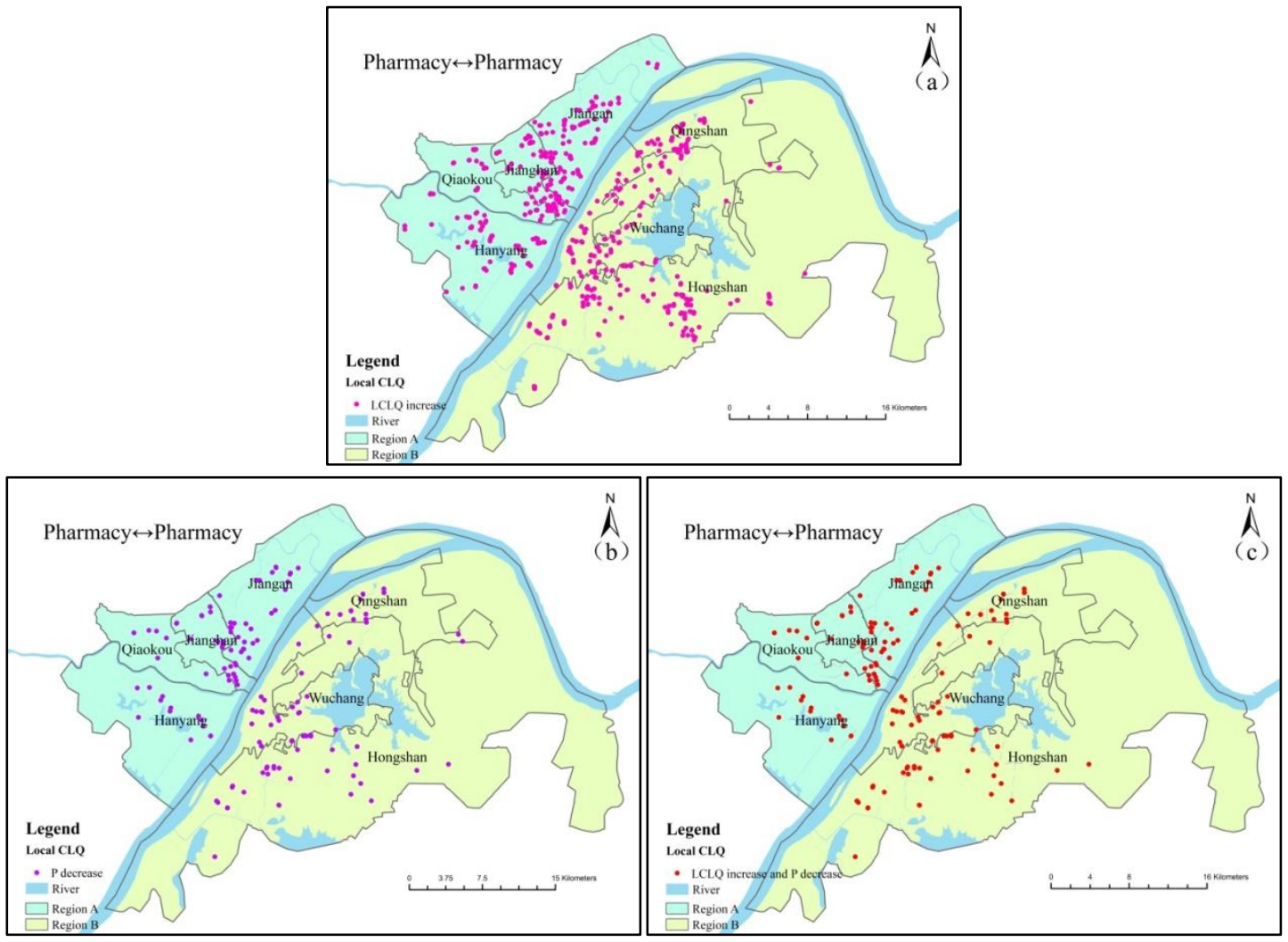

Figure 10. Spatial distribution of LCLQ and Pvalues for pharmacies after partition.

In short, the impact of the natural barrier (the Yangtze River) on the LCLQ analysis caused a significant increase in the directional spatial association between pharmacies. In terms of urban morphology, large-scale natural barriers in Wuhan, including the Yangtze River, Donghu and Nanhu lakes, have fragmented the road network and decreased transport accessibility between the two regions in the city. In the published literature, there have been long-standing debates and 
extensive evidence on the impacts of urban morphology on urban analyses, such as the impacts of urban form on commuting times and distances [63] and accessibility [64]. In particular, urban form has been shown to influence people's medical resource seeking behaviour [65]. The influence applies more to lower-level medical resources, such as pharmacies, clinics and community hospitals, that are visited more frequently for relatively minor health problems[66]. Patients with severe health problems were assumed to only visit higher-level hospitals [67], such as $3 \mathrm{~A}$ and specialist hospitals. The results have confirmed that the spatial form of the study area in this case bisection by the Yangtze River - did affect spatial associations between medical resources. It is suggested that a multi-scale analysis of LCLQ should be used for basic medical resources by splitting the study area into multiple parts that take account of natural barriers. Figure 11 shows a conceptualized influence pathway that reflects the impact of the spatial form of the study area on geographically-weighted co-location analysis. It suggests how natural barriers might influence behaviour and the scale of analysis required (Figure 11).

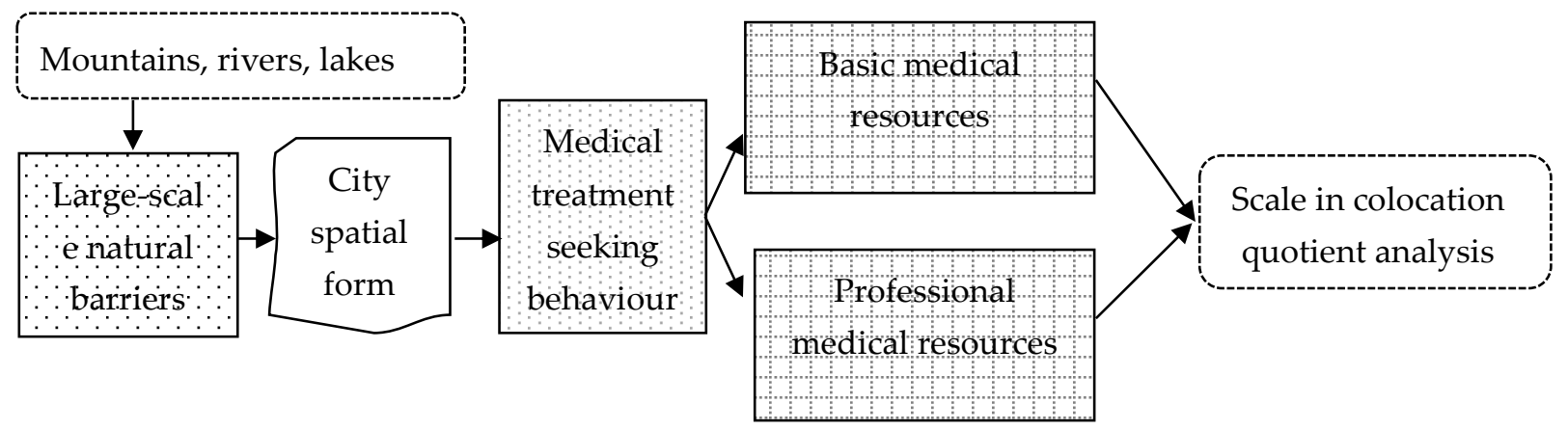

Figure 11. Conceptualising the impacts of spatial form on GWCLS analysis.

\section{Conclusions}

This paper has analysed the directional spatial associations between six medical resources across Wuhan City using a geographically weighted co-location quotient method. This approach is particularly useful for point categorical data. Using POI data from 2019, this study has generated the following empirical evidence:

(1) When taking the whole city as a single data set (i.e. without partition), 3A hospitals demonstrated the strongest spatial autocorrelation, suggestive of a clustering pattern across the city. This indicates the uneven distribution of 3A hospitcials at city level, with a single cluster of 3A hospitals located in Qingshan district. There were two networked communities of medical resources characterised by strong interactive co-locations within each system: firstly, pharmacies, clinics, and community hospitals (local services); and secondly, general, specialist and $3 \mathrm{~A}$ hospitals (city-level services). The spatial pattern of the two systems (basic and professional) reflects the history, investment and management of medical resources as well as the levels of technical, medical equipment and human resource specialism in Chinese cities [68, 69]. One strong symmetrical association between the two systems (community and general hospitals) was detected. This indicated the linkage between medical resources at local and city levels. The co-location association reflects the transition from local to city level hospitals in some cases [70]. Although the medical resources within each category (basic and professional) demonstrated a certain degree of spatial association, the two systems of medical resources were spatially mutually exclusive.

The health seeking behaviour for medical resources at the two levels (local vs city) was highly affected by the nature of the medical resource system. Basic medical resources (pharmacies, clinics and community hospitals) were most frequently visited at the local scale. Based on these results, using the whole city as a study area for LCLQ analysis is not advisable, especially if the city is split into multiple parts by large natural barriers. This paper demonstrated the impacts of 
the spatial form of a study area on the global and local values of LCLQ of local-level medical resources.

(2) When splitting the city into multiple data sets (e.g. regions A and B in this paper), the global and local LCLQ values for pharmacies, clinics and community hospitals changed signficantly in both regions after the spatial partition. The border areas between regions A and B were influenced most.

(3) The sensitivity analysis of LCLQ to study area spatial form in this paper has added a new spatial dimension into multi-scale GIS analysis. Cheng \& Fotheringham (2013) first proposed using multi-scale analysis after analysing the impact of country area on international comparative spatial analysis[21]. This paper focused on the impacts of the unique spatial form of the study area created by large-scale natural barriers. The multi-scale analysis in this paper has particular relevance to the analysis of urban resource patterns, such as medical resources, which might be accessed differently at local and city levels.

This study also proposes a multi-scale approach to bandwidths; varying the bandwidth size for different pairs of categories. Bandwidth size has been recognized as representative of scale in geographically weighted regression analysis [71]. In LCLQ analysis, there is no optimal bandwidth value for an adaptive bandwidth scheme. In this paper, different bandwidth sizes were examined for each pair of categories. For example, the best bandwidth size for measuring spatial association between pharmacies was 2, but increased to 4 for the spatial association between $3 \mathrm{~A}$ hospital and specialist hospitals.

This paper had some limitations. The POI data sets did not include all healthcare medical resources, such as moxibustion, foot therapy, massage, SPA or sweat steaming, which might form a third system of medical resources at the neighbourhood level. In this study the distance between points was measured using a direct-line Euclidean distance however in reality people's behaviour is more accurately predicted by transport distance, time and cost, and this is recommended as the measure of distance in future studies. Furthermore, the impacts of urban morphology on public service seeking behaviour should be evaluated in more detail.

Annotation:

LCLQ: local indicator of colocation quotient; GCLQ: Global indicator of colocation quotient.

\section{Consent for publication: Not applicable.}

Availability of data and materials: The location and classification of all medical resources within the study area were captured from Amap API (https://developer.amap.com/product/map) in December 2019 using a web crawling technique. District boundary and road network data were also collected from the National Basic Geographic Information System database. (http://bzdt.ch.mnr.gov.cn/index.html).A toolkit for measuring localized colocation quotient (LCLQ) is downloadable at http://seg.gmu.edu/clq (accessed on 30th August, 2021).

Competing interests: The authors declare that they have no competing interests.

Funding: This study was supported by Major Project of High Resolution Earth Observation System (grant no. GFZX0404130302); National Natural Science Foundation of China (grant no. 41871137; 41867071); Scientific Research Cultivation Project 2020 by Hubei University of Economics (grant no. PYYB202009).

Author Contributions: Q.C. wrote the whole paper and formal analysis. J.C. designed the research framework and revised the manuscript. J.T. collected the data and jointly analyzed.

\section{References}


1. Khodamoradi, A.; Hassanipour, S.; Daryabeygi-Khotbehsara, R.; Ahmadi, B., The trend of population aging and planning of health services for the elderly: A review study. Journal of Torbat Heydariyeh University of Medical Sciences 2019, 6, (3), 81-95.

2. $\quad$ Bureau, W. S., Wuhan Statistical Yearbook. Wuhan Statistical Yearbook: 2019.

3. Zhang, Z.; Yao, W.; Wang, Y.; Long, C.; Xinmiao, F. U., Wuhan and Hubei COVID-19 mortality analysis reveals the critical role of timely supply of medical resources. Journal of Infection 2020, 81, (1), $147-178$

4. Meng, Q.; Fang, H.; Liu, X.; Yuan, B.; Xu, J., Consolidating the social health insurance schemes in China: towards an equitable and efficient health system. The Lancet 2015, 386, (10002), 1484-1492.

5. Fan, X.; Su, M.; Si, Y.; Zhao, Y.; Zhou, Z., The benefits of an integrated social medical insurance for health services utilization in rural China: evidence from the China health and retirement longitudinal study. International Journal for Equity in Health 2021, 20, (1), 126.

6. Qian, Y.; Zhou, Z.; Yan, J. e.; Gao, J.; Wang, Y.; Yang, X.; Xu, Y.; Li, Y., An economy-related equity analysis of health service utilization by women in economically underdeveloped regions of western China. International Journal for Equity in Health 2017, 16, (1), 186.

7. Fu, X.; Sun, N.; Xu, F.; Li, J.; Tang, Q.; He, J.; Wang, D.; Sun, C., Influencing factors of inequity in health services utilization among the elderly in China. International Journal for Equity in Health 2018, 17, (1), 144.

8. $\quad$ Broome, M. R.; Woolley, J. B.; Tabraham, P.; Johns, L. C.; Bramon, E.; Murray, G. K.; Pariante, C.; McGuire, P. K.; Murray, R. M., Impact of Local Resources on Hospitalization Patterns of Medicare Beneficiaries and Propensity to Travel Outside Local Markets. Cosmo Publications 2010, 26, (1), 20-29.

9. Pra, B.; Zz, B.; Mpkc, D.; Yq, B., Evaluation of the spatial equity of medical facilities based on improved potential model and map service API: A case study in Zhengzhou, China. Applied Geography 2020, 119.

10. Tsutsui, A.; Taniyama, Y.; Ohno, Y., Driving to Childhood Cancer Hub Hospitals: A Study on Hospital Accessibility in Japan. Asian Pacific Journal of Cancer Prevention 2020, 21, (6), 1725-1730.

11. Cheng, L.; Yang, M.; Vos, J. D.; Witlox, F., Examining geographical accessibility to multi-tier hospital care services for the elderly: A focus on spatial equity. Journal of Transport \& Health 2020, 19.

12. Zhou, L.; Meng, F., Dynamics of an SIR epidemic model with limited medical resources revisited. Nonlinear Analysis Real World Applications 2012, 13, (1), 312-324.

13. Oshan, T. M.; Smith, J. P.; Fotheringham, A. S., Targeting the spatial context of obesity determinants via multiscale geographically weighted regression. International Journal of Health Geographics 2020, 19, (1), 11 .

14. Liu, T.; Yang, S.; Peng, R.; Huang, D., A Geographically Weighted Regression Model for Health Improvement: Insights from the Extension of Life Expectancy in China. Applied Sciences 2021, 11, (5).

15. Pius; Krütli; Thomas; Rosemann; Kjell; Y.; Trnblom; Timo; Smieszek, How to Fairly Allocate Scarce Medical Resources: Ethical Argumentation under Scrutiny by Health Professionals and Lay People. PLOS ONE 2016, 11, (7), 1-18.

16. Choi, Y.; Jeung, I. Y.; Park, J. J., Comparative Analysis of Spatial Impact of Living Social Overhead Capital on Housing Price by Residential type. KSCE Journal of Civil Engineering 2021, 25, (3), 1056-1065.

17. Casarrubias-Jaimez, A. I.; Ana Laura, J.-L.; Efraín, T.-S.; José Luis, R.-A.; Ramos-Quintana, F., Dealing with the Understanding of the Dynamics Related to Multifactorial Temporal Interactions That Spatially Affect the Landscape of Coastal Lagoons. Water 2021, 13, (15), 2099. 
18. Andresen, M.; Malleson, N., Spatial Heterogeneity in Crime Analysis. Crime Modeling and Mapping Using Geospatial Technologies 2013, 3-23.

19. Swift, A.; Liu, L.; Uber, J., Reducing MAUP bias of correlation statistics between water quality and GI illness. Computers, Environment and Urban Systems 2008, 32, (2), 134-148.

20. Xie, B.; An, Z.; Zheng, Y.; Li, Z., Healthy aging with parks: Association between park accessibility and the health status of older adults in urban China. Sustainable Cities and Society 2018, 43, 476-486.

21. Cheng, J.; Fotheringham, A. S., Multi-scale issues in cross-border comparative analysis. Geoforum 2013, 46, 138-148.

22. Cheng, J.; Masser, I., Urban growth pattern modeling: a case study of Wuhan city, PR China. Landscape and Urban Planning 2003, 62, (4), 199-217.

23. Chen, J.; Zhang, Y.; Yuanjian, Y. U., Effect of MAUP in Spatial Autocorrelation. Acta Geographica Sinica 2011, 66, (12), 1597-1606.

24. Swift, A.; Liu, L.; Uber, J., MAUP sensitivity analysis of ecological bias in health studies. Geojournal 2014, 79, (2), 137-153.

25. Budde, R.; Neumann, U., The size ranking of cities in Germany: caught by a MAUP? GeoJournal 2018, 26, (3), 118-133.

26. Jones, K.; Manley, D.; Johnston, R.; Owen, D., Modelling residential segregation as unevenness and clustering: A multilevel modelling approach incorporating spatial dependence and tackling the MAUP. Environment and Planning B: Urban Analytics and City Science 2018, 45, (6), 1122-1141.

27. He, Q.; He, W.; Song, Y.; Wu, J.; Yin, C.; Mou, Y., The impact of urban growth patterns on urban vitality in newly built-up areas based on an association rules analysis using geographical 'big data'. Land Use Policy 2018, 78, 726-738.

28. Huang, B.; Zhou, Y.; Li, Z.; Song, Y.; Cai, J.; Tu, W., Evaluating and characterizing urban vibrancy using spatial big data: Shanghai as a case study. Environment and Planning B: Urban Analytics and City Science 2019, 47, (9), 1543-1559.

29. Milias, V.; Psyllidis, A., Assessing the influence of point-of-interest features on the classification of place categories. Computers, Environment and Urban Systems 2021, 86, 101597.

30. Zhang, Y.; Cheng, H.; Huang, D.; Fu, C., High Temporal Resolution Land Use Regression Models with POI Characteristics of the PM2.5 Distribution in Beijing, China. International Journal of Environmental Research and Public Health 2021, 18, (11), 6143.

31. Chen, H.; Song, X.; Xu, Changhui; Zhang, X., Using Mobile Phone Data to Examine Point-of-Interest Urban Mobility. Journal of Urban Technology 2021, 27, (4), 1-16.

32. Cromley, R. G.; Hanink, D. M.; Bentley, G. C., Geographically Weighted Colocation Quotients: Specification and Application. Professional Geographer 2014, 66, (1), 138-148.

33. Yue, H.; Zhu, X.; Ye, X.; Guo, W., The Local Colocation Patterns of Crime and Land-Use Features in Wuhan, China. International Journal of Geo-Information 2017, 6, (10), 307.

34. Leslie, T. F.; Kronenfeld, B. J., The Colocation Quotient: A New Measure of Spatial Association Between Categorical Subsets of Points. Geographical Analysis 2011, 43, (3), 306-326.

35. Leslie, T. F.; Frankenfeld, C. L.; Makara, M. A., The spatial food environment of the DC metropolitan area: Clustering, co-location, and categorical differentiation. Applied Geography 2012, 35, (1-2).

36. Nilsson, I. M.; Smirnov, O. A., Measuring the effect of transportation infrastructure on retail firm co-location patterns. Journal of Transport Geography 2016, 51, 110-118. 
37. Wang, F.; Hu, Y.; Wang, S.; Li, X., Local indicator of colocation quotient with a statistical significance test: examining spatial association of crime and facilities. The Professional Geographer 2017, 69, (1), 22-31.

38. Hu, Y.; Zhang, Y.; Shelton, K. S., Where are the dangerous intersections for pedestrians and cyclists: A colocation-based approach. Transportation Research Part C: Emerging Technologies 2018, 95, 431-441.

39. Zx, A.; Hao, L. A.; Yc, A.; Wy, B., Detecting urban fire high-risk regions using colocation pattern measures - ScienceDirect. Sustainable Cities and Society 2019, 49, 8.

40. Kuo, P.-F.; Lord, D., Applying the colocation quotient index to crash severity analyses. Accident Analysis \& Prevention 2020, 135, 105368.

41. Yu, Q.; Gu, Y.; Yang, S.; Zhou, M., Discovering Spatiotemporal Patterns and Urban Facilities Determinants of Cycling Activities in Beijing. Journal of Geovisualization and Spatial Analysis 2021, 5, (1), 16 .

42. Wuhan Bureau of Statistics, Wuhan Statistical Yearbook. 2020. Wuhan Statistical Yearbook. 2020: 2020 .

43. Ye, H., Research on the development of TCM medical service in China under the background of "Healthy China 2030". Journal of Traditional Chinese Medicine Management 2021, 29, (12), 226-227.

44. Luo, B.; Xia, W.; Lu, J. e.; Wei, Q., Exploration and Research on health industry planning oriented by implementation -- A case study of Wuhan. Research on modern City 2020, (04), 52-59.

45. Chuansheng, G.; Zhen, L., Effect and trend of the policy of graded diagnosis and treatment at high quality development stage. International Journal of Environmental Research \& Public Health 2019, (11), 65-72.

46. Liu, G. G.; Shi, J.; Wang, X.; Yang, H.; Zhu, H., Does Ownership Matter for Medical System Performance? Evidence From a Natural Experiment in Suqian, China. INQUIRY: The Journal of Health Care Organization, Provision, and Financing 2020, 57, 0046958020944338.

47. Av, A.; Bp, A.; Am, B.; Eg, B., Unmet medical needs, resources allocation and issues regarding cost-effectiveness in critical care. Anaesthesia Critical Care \& Pain Medicine 2020, 39, (1), 111-112.

48. Li, Y.; Hu, J.; Gao, Z. X., Research on Chinese healthcare big data resource catalog system and technical framework. Chinese Journal of Health Information management 2019, 016, (003), 249-256.

49. Zhou, Z.; Zhao, Y.; Shen, C.; Lai, S.; Nawaz, R.; Gao, J., Evaluating the effect of hierarchical medical system on health seeking behavior: A difference-in-differences analysis in China. Social Science \& Medicine 2021, 268, 113372.

50. An, H.; Xiao, C.; Ding, M., The Spatial Pattern of Ski Areas and Its Driving Factors in China: A Strategy for Healthy Development of the Ski Industry. Sustainability 2019, 11, (11), 3138.

51. Xiong, X.; Zhang, Z.; Ren, J.; Zhang, J.; Pan, X.; Zhang, L.; Gong, S.; Jin, S., Impact of universal medical insurance system on the accessibility of medical service supply and affordability of patients in China. PLoS One 2018, 13, (3), e0193273.

52. Zheng, D.; Gong, J.; Zhang, C., Efficiency of medical service systems in the rural areas of Mainland China: a comparative study from 2013 to 2017. Public Health 2019, 171, 139-147.

53. Yuan, B.; Jian, W.; Martinez-Alvarez, M.; McKee, M.; Balabanova, D., Health system reforms in China a half century apart: Continuity but adaptation. Social Science \& Medicine 2020, 265, 113421.

54. Zhu, K.; Zhang, L.; Yuan, S.; Zhang, X.; Zhang, Z., Health financing and integration of urban and rural residents' basic medical insurance systems in China. International Journal for Equity in Health 2017, 16, (1), 194. 
55. Pan, X.-F.; Xu, J.; Meng, Q., Integrating social health insurance systems in China. The Lancet 2016, 387, (10025), 1274-1275.

56. Brooks, J. M.; Doucette, W. R.; Wan, S.; Klepser, D. G., Retail Pharmacy Market Structure and Performance. INQUIRY: The Journal of Health Care Organization, Provision, and Financing 2008, 45, (1), 75-88.

57. Chen, J., The Effects of Competition on Prescription Payments in Retail Pharmacy Markets. Southern Economic Journal 2019, 85, (3), 865-898.

58. Ren, P.; Xu, Z.; Liao, H.; Zeng, X.-J., A thermodynamic method of intuitionistic fuzzy MCDM to assist the hierarchical medical system in China. Information Sciences 2017, 420, 490-504.

59. Yuan, J., Application of Reimbursement Model DingTalk of Scientific Research Funds in a Public Hospital in Wuhan City. Medicine and Society 2019, (3), 56-66.

60. Chen, J.; Yu, H.; Dong, H., Effect of the new rural cooperative medical system on farmers' medical service needs and utilization in Ningbo, China. BMC Health Services Research 2016, 16, (1), 593.

61. Lei, X.; Jiang, H.; Liu, C.; Ferrier, A.; Mugavin, J., Self-Medication Practice and Associated Factors among Residents in Wuhan, China. International Journal of Environmental Research and Public Health 2018, 15, (1), 68 .

62. Huang, Y.; Yao, D.; Xi, X.; Wang, Y.; Yao, W., Current status of pharmacy services in primary healthcare institutions in Jiangsu Province, China. Australian Journal of Primary Health 2020, 26, (5), 424-430.

63. Engelfriet, L.; Koomen, E., The impact of urban form on commuting in large Chinese cities. Transportation 2018, 45, (5), 1269-1295.

64. Du, F.; Mao, L.; Wang, J.; Jin, H., Inferring transit-based health seeking patterns from smart card data A case study in Beijing, China. Health \& Place 2020, 65, 102405

65. Jia, L., Evaluation and Analysis of Medical and Health Resource Allocation Efficiency in HuBei Province. E3S Web of Conferences 2020, 214.

66. Brambilla; Andrea; Capolongo; Stefano, Healthy and Sustainable Hospital Evaluation-A Review of POE Tools for Hospital Assessment in an Evidence-Based Design Framework. Buildings 2019, 9, (4), 76-76.

67. Lei; Zhu; Shuang; Zhong; Wei; Tu; Jing; Zheng; Shenjing; He, Assessing Spatial Accessibility to Medical Resources at the Community Level in Shenzhen, China. International Journal of Environmental Research \& Public Health 2019.

68. Duan, J.; Jiao, F.; Zhang, Q.; Lin, Z., Predicting Urban Medical Services Demand in China: An Improved Grey Markov Chain Model by Taylor Approximation. International Journal of Environmental Research and Public Health 2017, 14, (8).

69. Guo, B.; Xie, X.; Wu, Q.; Zhang, X.; Cheng, H.; Tao, S.; Quan, H., Inequality in the health services utilization in rural and urban china: A horizontal inequality analysis. Medicine (Baltimore) 2020, 99, (2), $18625-18625$.

70. Qiu, Y.; Lu, W.; Guo, J.; Sun, C.; Liu, X., Examining the Urban and Rural Healthcare Progress in Big Cities of China: Analysis of Monitoring Data in Dalian from 2008 to 2017. International Journal of Environmental Research and Public Health 2020, 17, (4).

71. Fotheringham, A. S.; Brunsdon, C.; Charlton, M., Geographically weighted regression: the analysis of spatially varying relationships. John Wiley \& Sons: 2002. 